\title{
Shear-Driven Flow Ice Chromatography as a Possible Tool Probing Ice/Water Interface
}

\author{
Masaya Shimizu, Makoto Harada, Akihide Hibara, and Tetsuo OKada ${ }^{\dagger}$ \\ Department of Chemistry, Tokyo Institute of Technology, Meguro, Tokyo 152-8551, Japan
}

\begin{abstract}
Although ice chromatography is a useful probe of ice interfaces, its low separation efficiency has often made difficult to access the ice/water interface. Coupling of this method with shear-driven flow chromatography, which has high separation potential, solves the problems involved in ice chromatography. This paper reports on shear-driven flow ice chromatographic instrumentation, and discusses the separation performance. Electrostatic separation of positively and negatively charged dyes is demonstrated with an $\mathrm{OH}^{-}$-doped ice plate as a stationary phase.
\end{abstract}

Keywords Shear flow, ice chromatography, electrostatic interaction at ice interface, interface between ice and aqueous solution

(Received March 8, 2016; Accepted March 23, 2016; Published July 10, 2016)

\section{Introduction}

Ice chromatography, in which ice particles are used as a liquid chromatographic stationary phase, has been used for separation based on adsorption through hydrogen bonding between solutes and the ice surface, ${ }^{1-3}$ retention control by adsorption/partition switching, ${ }^{4,5}$ and chiral separation. ${ }^{6,7}$ This method also provides information on phenomena occurring at the interface between the ice and a mobile phase. ${ }^{8}$ A water-immiscible organic mobile phase, such as hexane, has been used to prevent dissolution of the ice stationary phase. Ice chromatographic probing has therefore been restricted to ice/organic solvent interfaces. In addition, the separation performance of ice chromatography is not very high, mainly because of the sintering of ice particles progressed during chromatographic runs. ${ }^{9,10}$ The instability of an ice interface should be more serious in aqueous environments. No efficient applications of aqueous mobile phases in ice chromatography have been reported so far for this reason. Because various reactions and phenomena occur at the ice/water interface in the natural environment, ${ }^{11}$ ice chromatography could be an efficient probe to this interface.

A mobile phase is fed to a separation column by pressurized flow in usual liquid chromatography. Fine stationary phase particles, which allow a reduction of the time required for radial diffusion of a solute, are often employed to gain a high separation performance. ${ }^{12,13}$ Desmet et al. ${ }^{14-18}$ devised sheardriven flow chromatography, in which a mobile phase is sandwiched between two plates and the movement of one wall causes a shear-driven flow of the mobile phase. In their study, the surface of a fixed wall was modified to act as a stationary phase. A high separation performance and high speed separation over the usual packed columns were predicted, albeit the method has not been well recognized because of the complicated

† To whom correspondence should be addressed.

E-mail: tokada@chem.titech.ac.jp experimental system.

In the present paper, we demonstrate instrumentation and preliminary results of shear-driven flow ice chromatography. The stationary phase in the present method is an ice plate. Therefore, it is a key technique to prepare the ice stationary phase and to keep it stable during chromatographic runs. Unlike packed columns, the sintering of ice does not occur on a single ice plate. In addition, the use of an aqueous mobile phase is easier in this configuration than in a packed column.

\section{Experimental}

A schematic diagram of the present experimental setup is shown in Fig. S1. The movement of solutes was detected with a fluorescence microscope (Olympus Model BX-41) equipped with $\mathrm{a} \times 1.25$ objective $(\mathrm{N} / \mathrm{A}=0.04)$. The light source was an Hg-lamp. The excitation $\left(\lambda_{\mathrm{ex}}\right)$ and detection $\left(\lambda_{\mathrm{ex}}\right)$ wavelengths were set to $\lambda_{\mathrm{ex}}=460-495 \mathrm{~nm}$ and $\lambda_{\mathrm{em}}>510 \mathrm{~nm}$ for fluorescein (FL) and $\lambda_{\mathrm{ex}}=530-550 \mathrm{~nm}$ and $\lambda_{\mathrm{em}}>575 \mathrm{~nm}$ for Rhodamine B (RB). These fluorescent dyes were purchased from Wako Pure Chemicals and used as received. The temperature of the ice stationary phase was controlled with a Peltier array, which was driven by a Peltier controller (Model TDC-2020R, Cell System). The reversed side of the Peltier array was cooled by a chiller. The moving wall was an OHP sheet $(40 \times 297 \mathrm{~mm})$, on which lines were printed with a laser printer. These printed lines acted as channel side walls. The height of the lines was measured by a laser displacement meter, ranging over $6-10 \mu \mathrm{m}$. The cross-section of the separation channel was $10 \mathrm{~mm}$ in width, $20 \mathrm{~mm}$ in length, and several micrometers in height. The working channel height will be evaluated based on the peak broadening, as discussed later. The OHP sheet was pasted on a Plexiglas plate with an open window, in which a $\mathrm{Cu}$ plate $(40 \times$ $90 \mathrm{~mm}$ ) was fitted. The Plexiglas plate was set on the Peltier array put on a microscope stage. The $\mathrm{Cu}$ plate was always in contact with the Peltier array. The tip of the OHP sheet was 
fixed on an X-stage, which pulled the OHP sheet at a constant rate. Water or an aqueous solution was poured in $\mathrm{Cu}^{-}$frame (inner size, $20 \times 20 \mathrm{~mm}$ ), and was frozen on another Peltier array at $-5.0^{\circ} \mathrm{C}$. Slow freezing allowed the preparation of a transparent ice plate. The thickness of the ice plate was $1 \mathrm{~mm}$. A small amount of an appropriate aqueous phase was put on the OHP sheet set on the Peltier array kept at $-2.0^{\circ} \mathrm{C}$. The ice plate was transferred together with the $\mathrm{Cu}^{-}$frame onto the $\mathrm{OHP}$ sheet. Ice chromatographic separation was carried out on the $\mathrm{Cu}$ plate fitted to the Plexiglas plate. The temperature of the separation channel was maintained by thermal conduction through the $\mathrm{Cu}$ plate. The aqueous phase was spread in the space between the OHP sheet and the ice plate. Any unnecessary aqueous phase was then removed.

A sample solution of several hundreds of $\mathrm{pL}$ (smaller than $1 \mathrm{~nL}$ ) was put on the OHP sheet, $5 \mathrm{~mm}$ apart from the edge of the ice plate with a piezo devise (PIJD-1, Wave Guilder). The sample was introduced in the separation channel by the movement of the OHP sheet. An additional mobile phase was put on the OHP sheet just behind the sample solution; after sample injection, the mobile phase was fed into the separation channel. In some cases, air bubbles were introduced in the channel upon injecting a sample, which interfered with the subsequent separation. This was avoided by reducing the distance between the sample spot and the additional mobile phase. When air bubbles were observed in the channel, the channel was cleaned, and all of the above procedure was repeated. Microscopic observations were carried out through a transparent ice plate. The space under the microscopy was covered and purged by cooled dry nitrogen to prevent frost adhesion and melting of ice. Since the temperature of nitrogen was not controlled, the temperature at the top of the ice plate was $1-1.5^{\circ} \mathrm{C}$ higher than the Peltier temperature. A slide glass instead of the ice plate was used to study a shear-driven flow in the channel.

\section{Results and Discussion}

Unless slipping occurs on the wall, the linear flow rate of a streamline on the fixed wall is zero, while that on the moving wall is equal to that of the moving rate of the wall $\left(v_{\text {wall }}\right)$. The solution of Navier-Stokes equation in the absence of a pressure gradient is given by ${ }^{19}$

$$
v_{\mathrm{x}(\mathrm{z})}=v_{\mathrm{wall}} z / d \text {. }
$$

where $v_{\mathrm{x}(\mathrm{z})}$ is the linear flow rate in the axial $(x)$ direction at $z$, and $d$ is the thickness of the channel. This flow profile is known as Couette flow. The flow rate of a streamline in the channel is thus expected to be linearly changed between two walls. According to this flow profile, the average linear flow rate $\left(v_{\mathrm{av}}\right)$ is equal to half of the maximum linear flow rate, which is the equal to $v_{\text {wall }}$; thus, $v_{\mathrm{av}}=0.5 v_{\text {wall }}$.

Typical fluorescence microscopic images of FL in the sheardriven $\left(v_{\text {wall }}=0.79 \mathrm{~mm} \mathrm{~s}^{-1}\right)$ flow are shown in Fig. 1A. From these images, the travel rate of FL $\left(v_{\mathrm{FL}}\right)$ is determined to be $0.4 \mathrm{~mm} \mathrm{~s}^{-1}$. The diffusion coefficient of FL $(D)$ is reported as $4.2 \times 10^{-10} \mathrm{~m}^{2} \mathrm{~s}^{-1} .^{20}$ The diffusion time of FL over the entire channel height is $0.12 \mathrm{~s}$, assuming $d=10 \mu \mathrm{m}$. Thus, the distribution of FL in the $z$-direction is uniform, and therefore $v_{\mathrm{FL}}=v_{\mathrm{av}}$. The average flow rates were measured from FL images by varying $v_{\text {wall }}$ to assess the applicability of the Couette flow in the present experimental system. Figure 1B shows the dependence of $v_{\mathrm{av}}=v_{\mathrm{FL}}$ on $v_{\mathrm{wall}}$ observed in the channel formed
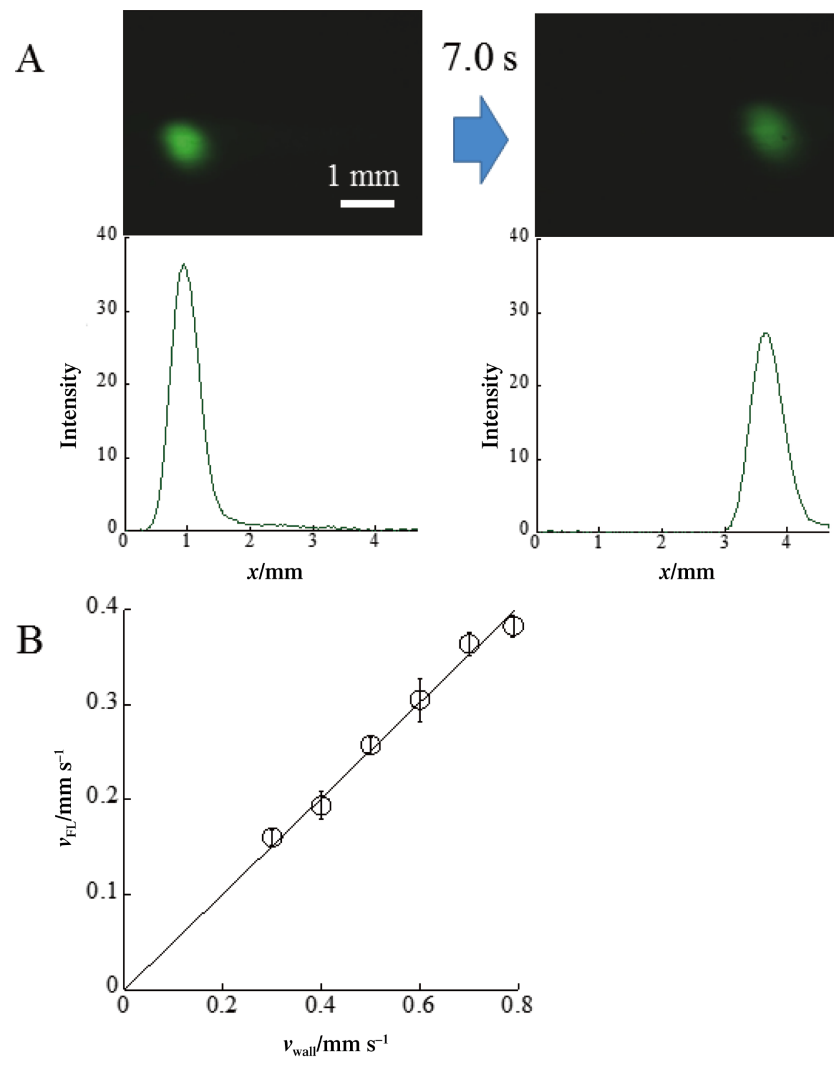

Fig. 1 (A) Fluorescence micrographs and intensities along the flow (x) axis for FL traveling in a separation channel. The time interval between two images was $7.0 \mathrm{~s}$. Fixed wall, glass slide. Mobile phase, water. $v_{\text {wall }}=0.39 \mathrm{~mm} \mathrm{~s}^{-1}$. (B) Relation between $v_{\mathrm{FL}}$ and $v_{\text {wall }}$.

between the OHP moving wall and the fixed glass wall; $v_{\mathrm{av}}$ is obviously proportional to $v_{\text {wall }}$. The slope of the plot is equal to 0.504, suggesting the establishment of Couette flow in the channel.

Desmet et al. derived an equation representing $\operatorname{HETP}(H)$ for a rectangular-shaped channel with a finite lateral width for shear-driven flow chromatography. ${ }^{16}$ When no retention occurs, the equation is simplified to

$$
H=\frac{2 D}{v_{\mathrm{av}}}+\frac{3.48 v_{\mathrm{av}} d^{2}}{30 D}
$$

Equation (2) clearly indicates that $d$ should be as small as possible to maintain high separation performance. For FL, the dependence of $H$ on $v_{\text {av }}$ can be calculated for several $d$ values as shown in Fig. S2. The minimum H occurs: e.g. $H=4.8 \mu \mathrm{m}$ at $v_{\mathrm{av}}=0.34 \mathrm{~mm} \mathrm{~s}^{-1}$ for $d=5 \mu \mathrm{m} ; H=9.6 \mu \mathrm{m}$ at $v_{\mathrm{av}}=0.17 \mathrm{~mm} \mathrm{~s}^{-1}$ for $d=10 \mu \mathrm{m}$. Under the optimum condition, the ideal theoretical number is estimated to be $2.1 \times 10^{3} \mathrm{~cm}^{-1}$ for $d=$ $5 \mu \mathrm{m}$ and $1.0 \times 10^{3} \mathrm{~cm}^{-1}$ for $d=10 \mu \mathrm{m}$.

Another important factor causing sample band spreading is injection. In the present study, the sample volume was kept to be as small as $1 \mathrm{~nL}$. Under the ideal condition, the length of a sample zone is estimated to be $350 \mu \mathrm{m}$, assuming an isotropically spread sample zone. The actual sample zone width was $470 \pm 110 \mu \mathrm{m}(n=4)$. The efficient theoretical plate number $\left(N_{\text {eff }}\right)$ is given by ${ }^{18}$ 


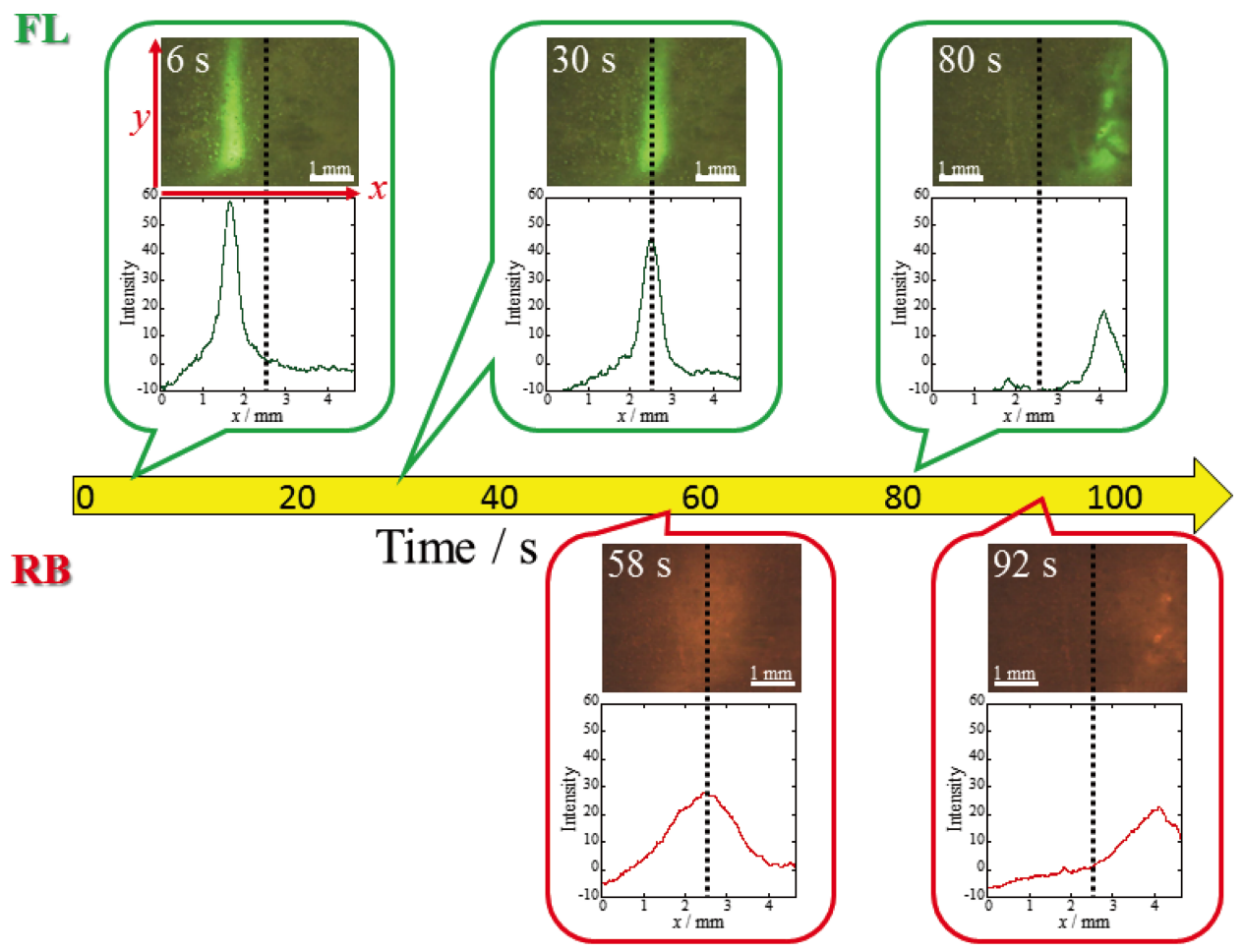

Fig. 2 Micrographic snapshots and fluorescence intensities along the flow axis for simultaneously injected FL and RB in shear-driven flow ice chromatography. Fixed wall, $1.0 \mathrm{mM} \mathrm{NaOH}$-doped ice. Mobile phase, $1 \mathrm{mM} \mathrm{NaCl}+0.1 \mathrm{mM} \mathrm{NaOH}$. $v_{\text {wall }}=0.06 \mathrm{~mm} \mathrm{~s}^{-1}$. Temperature, $-2^{\circ} \mathrm{C}$.

$$
N_{\text {eff }}=\frac{L^{2}}{\sigma^{2}}=\frac{L^{2}}{\sigma_{\text {inj }}{ }^{2}+\sigma_{\mathrm{ch}}{ }^{2}}=\frac{L^{2}}{\frac{w_{\text {inj }}^{2}}{12}+H L}
$$

where $L$ is the channel length, $\sigma_{\mathrm{inj}}{ }^{2}$ and $\sigma_{\mathrm{ch}}{ }^{2}$ are the variances coming from injection and the passage though the channel before detection, respectively, and $w_{\text {inj }}$ is the width of the sample zone. Equation (3) predicts that $N_{\text {eff }}=1.5 \times 10^{3} \mathrm{~cm}^{-1}$ for $d=5 \mu \mathrm{m}$ and $N_{\text {eff }}=8.7 \times 10^{2} \mathrm{~cm}^{-1}$ for $d=10 \mu \mathrm{m}$, which are ca. 28 and $13 \%$ smaller than the corresponding $N$ estimated in the absence of the peak broadening by injection, respectively. The present sample injection scheme does not severely impair the separation performance.

Based on the band broadening of FL, the channel height can be evaluated. Two chromatograms are shown in Fig. 1A. The peak width $(w)$ increases from 0.92 to $1.07 \mathrm{~mm}$ for the interval between two measurements $\left(7 \mathrm{~s}\right.$ at $\left.v_{\mathrm{av}}=0.40 \mathrm{~mm} \mathrm{~s}^{-1}\right)$. From Eq. (3), the following relation can be derived:

$$
\Delta w^{2}=12 H \Delta L
$$

where $\Delta w^{2}$ and $\Delta L$ are the differences in $w^{2}$ between two peaks and in the distance travelled during the measurements, respectively. $H$ is estimated to be $4.5 \mu \mathrm{m}$ from these values. This situation is established for $d=4.7 \mu \mathrm{m}$. Thus, the channel height can be estimated from the two peaks of a solute, which are measured at different moments.

The surface of ice is negatively charged by the dissociation of $\mathrm{H}^{+}$from the $\mathrm{OH}$ dangling bonds. ${ }^{10}$ Also, when an aqueous $\mathrm{NaCl}$ is frozen, $\mathrm{Cl}^{-}$is more preferably entrapped in the ice crystal than $\mathrm{Na}^{+} .{ }^{21}$ Although the charge imbalance is relaxed by the transfer of $\mathrm{OH}^{-}$and $\mathrm{H}^{+}$to the solution and ice phases, respectively, the ice phase is likely to be negatively charged in frozen aqueous
$\mathrm{NaCl}$. Zeta potential measurements at the ice/aqueous solution interface were attempted, and the point of zero charge of ice was reported as being $\mathrm{pH} 3.5$ in a $1 \mathrm{mM} \mathrm{NaCl}$ solution..$^{22}$ If the ice surface is actually negatively charged, cationic solutes should be electrostatically attracted by the ice fixed wall. The separation of positively charged RB and negatively charged FL was studied with the present apparatus. The result is given in Fig. S3. In the present detection set-up, the mirror units for detection were manually switched between two types appropriate for FL and RB detections. Therefore, the times are not synchronized for FL and RB detection. No separation between FL and RB was confirmed with the pure ice fixed wall. Larger band broadening is seen in this case than for a glass stationary plate, possibly due to the poor flatness of the ice wall.

When $\mathrm{OH}^{-}$is doped in ice (in other word, frozen aqueous $\mathrm{NaOH}), \mathrm{OH}^{-}$is partially entrapped in the ice structure. Doping of $\mathrm{OH}^{-}$produces the Bjerrum L-defects, and negative charges emerge on the ice surface. ${ }^{10} \mathrm{NaOH}$-doped ice was employed as a fixed wall because it is expected to more strongly attract RB than the pure ice wall. Figure 2 shows snapshots at several moments taken for FL and RB. The corresponding fluorescence intensity scans along the flow axis are also given in the figure. Obviously, the migration of RB is retarded compared with FL, implying that the enhanced negative charges on the ice fixed wall effectively work for RB retention. The band spreading for RB more severely occurs than that for FL. Large band spreading for RB was always observed even with a glass wall. RB is likely to form aggregates in an aqueous solution. The slow diffusion of molecular aggregates possibly causes large band broadening. ${ }^{23,24}$ At relatively high $v_{\mathrm{av}}, H$ is determined dominantly by the second term of Eq. (2), which comes from the inhomogeneous distribution of a solute in the direction orthogonal to the flow direction. If $D$ is one order of magnitude 


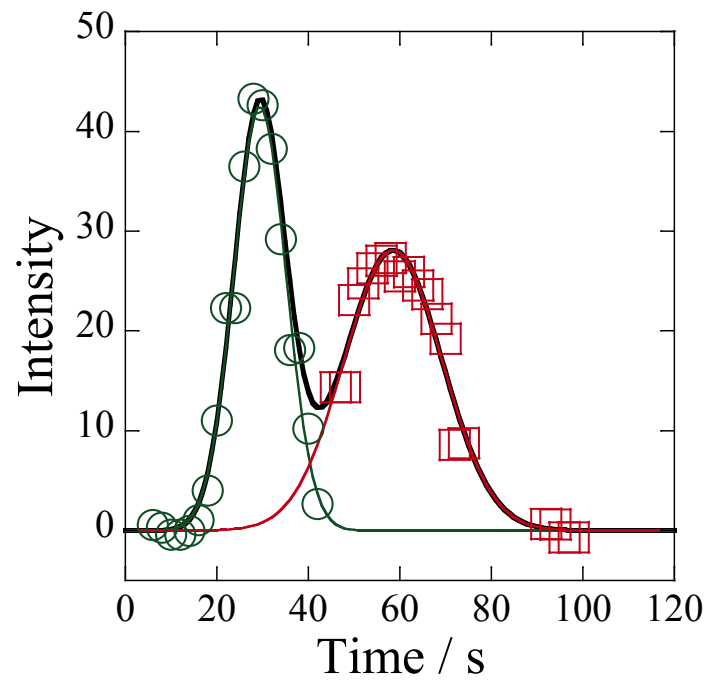

Fig. 3 Shear-driven flow ice chromatogram of FL and RB. Fluorescence was detected at $x=2.5 \mathrm{~mm}$ shown by broken lines in Fig. 2 for FL (green circles) and RB (red squares). Gaussian curves are for eye guide.

smaller for aggregates than the intrinsic value, $H$ is ten-times larger and the peak width is three times larger than in the absence of aggregation.

From the peak widths of FL, the channel height can be estimated as discussed above. For the first two peaks depicted in Fig. 2, the peak widths are 0.72 and $0.88 \mathrm{~mm}$, respectively. Taking $v_{\mathrm{av}}=0.03 \mathrm{~mm} \mathrm{~s}^{-1}$ into account, $H$ is estimated to be $30 \mu \mathrm{m}$. These values are provided when the channel height is $14 \mu \mathrm{m}$. This value is almost three times as large as that calculated for the glass fixed wall, implying the poor flatness of the ice plate. It should be noted that the average linear flow rate is constant, even when the channel height is not uniform, because it solely depends on the moving rate of the OHP sheet. However, the volume flow rate depends on the location in the channel. This may cause turbulence of a flow and eventually result in band broadening. Figure 3 shows time changes of the fluorescence intensity monitored at $x=2.5 \mathrm{~mm}$ from the edge of the microscopic view. This chromatogram obviously demonstrates that FL and RB are separated by shear-driven flow ice chromatography.

In conclusion, a shear-driven flow ice chromatographic system was constructed; the separation of cationic and anionic dyes are separated by the electrostatic interaction with a negatively charged ice wall. Because of difficulty to prepare an ice plate with a flat surface, the flow in the channel may be perturbed. Also, the channel height is not small enough to obtain a high separation performance. However, a theoretical plate number, i.e. more than $1000 \mathrm{~cm}^{-1}$, can be attained even with the simple experimental setup employed in this work, if the fixed wall has a flat surface like a slide glass. The preparation of flat ice plates is thus a key technique in the application of this method to various separations that take place at the water/ice interface. Unwelcome roughness of the ice plate surface is caused when it is detached from a Peltier array. Therefore, an appropriate treatment of the surface of the Peltier array (attached $\mathrm{Cu}$ plate) should be studied to reduce the ice adhesion with the cryodevice surface. Studies of anti-freeze proteins in shear-driven flow ice chromatography, for example, should be of scientific interest because their detailed molecular behavior at the water/ ice interface, which is still under debate, may be revealed by this approach. ${ }^{25}$

\section{Acknowledgements}

This work was supported by a Grant-in-aid for Scientific Research from the Japan Society for Promotion of Science. The authors thank Prof. Desmet for his helpful discussions on this work.

\section{References}

1. T. Okada and Y. Tasaki, Anal. Bioanal. Chem., 2010, 396, 221.

2. Y. Tasaki and T. Okada, Anal. Chem., 2006, 78, 4155.

3. Y. Tasaki and T. Okada, J. Chromatogr. A, 2008, 1189, 72.

4. Y. Tasaki and T. Okada, Anal. Sci., 2009, 25, 177.

5. Y. Tasaki and T. Okada, Anal. Chem., 2011, 83, 9593.

6. T. Shamoto, Y. Tasaki, and T. Okada, J. Am. Chem. Soc., 2010, 132, 13135.

7. S. Takahashi, M. Harada, and T. Okada, Anal. Methods, 2016, 8, 105.

8. Y. Tasaki and T. Okada, J. Phys. Chem. C, 2008, 112, 2618.

9. J. R. Blackford, J. Phys. D: Appl. Phys., 2007, 40, R355.

10. V. F. Petrenko and R. W. Whitworth, "Physics of Ice", 1999, Oxford University Press, New York.

11. T. Bartels-Rausch, H.-W. Jacobi, T. F. Kahan, J. L. Thomas, E. S. Thomson, J. P. D. Abbatt, M. Ammann, J. R. Blackford, H. Bluhm, C. Boxe, F. Domine, M. M. Frey, I. Gladich, M. I. Guzmán, D. Heger, T. Huthwelker, P. Klán, W. F. Kuhs, M. H. Kuo, S. Maus, S. G. Moussa, V. F. McNeil , J. T. Newberg, J. B. C. Pettersson, M. Roeselo, and J. R. Sadeau, Atmos. Chem. Phys. Discuss., 2012, 12, 30409.

12. J. E. MacNair, K. C. Lewis, and J. W. Jorgenson, Anal. Chem., 1997, 69, 983.

13. B. Wei, B. J. Rogers, and M. J. Wirth, J. Am. Chem. Soc., 2012, 134, 10780.

14. D. Clicq, K. Pappaert, S. Vankrunkelsven, N. Vervoort, G. V. Baron, and G. Desmet, Anal. Chem., 2004, 76, 431A.

15. W. De Malsche, D. Clicq, H. Eghbali, V. Fekete, H. Gardeniers, and G. Desmet, Lab. Chip, 2006, 6, 1322.

16. G. Desmet and G. V. Baron, J. Chromatogr. A, 1999, 855, 57.

17. G. Desmet, N. Vervoort, D. Clicq, and G. V. Baron, J. Chromatogr. A, 2001, 924, 111.

18. G. Desmet, N. Vervoort, D. Clicq, A. Huau, P. Gzil, and G. V. Baron, J. Chromatogr. A, 2002, 948, 19.

19. B. R. Munson, T. H. Okiishi, W. W. Huebsch, and A. P. Rohmayer, "Fundamentals of Fluid Mechanics", 7th ed., 2013, John Wiley \& Sons, New York.

20. C. T. Culbertson, S. C. Jacobson, and J. M. Ramsey, Talanta, 2002, 56, 365.

21. H. Watanabe, T. Otsuka, M. Harada, and T. Okada, J. Phys. Chem. C, 2014, 118, 15723.

22. J. Drzymala, Z. Sadowski, L. Holysz, and E. Chibowski, J. Colloid Interface Sci., 1999, 220, 229.

23. M. K. Goftar, K. Moradi, and N. M. Kor, Eur. J. Exp. Biol., 2014, 4, 72.

24. N. O. Mchedlov-Petrosyan and Y. V. Kholin, Russ. J. Appl. Chem., 2004, 77, 414

25. J. G. Duman, Annu. Rev. Physiol., 2001, 63, 327. 